\title{
Progression of eye disease in "cured" leprosy patients: implications for understanding the pathophysiology of ocular disease and for addressing eyecare needs
}

\author{
Susan Lewallen, Narong C Tungpakorn, Sung-Hwa Kim, Paul Courtright
}

\begin{abstract}
Background-Ocular damage in leprosy is due either to nerve damage or infiltration by mycobacteria. There is currently little information about the magnitude and nature of incident ocular pathology in cured leprosy patients. This information would increase our understanding of the pathophysiology of ocular involvement in leprosy and help in developing programmes to address the eyecare needs of leprosy patients who have been released from treatment. The cumulative incidence of leprosy related ocular pathology and cataract was measured during an 11 year follow up period in cured leprosy patients released from treatment in Korea.

Methods-In 1988 standardised eye examinations were performed on 501 patients in eight resettlement villages in central South Korea. In May 1999 standardised eye examinations were repeated in this population.
\end{abstract}

Results-Among the patients in whom there was no sight threatening leprosy related ocular disease (lagophthalmos, posterior synechia, or keratitis) in 1988, $14.7 \%$ developed one or more of these conditions. Overall, among those with no vision reducing cataract in $1988,26.4 \%$ had developed a vision reducing lens opacity in at least one eye. Among patients examined in both 1988 and 1999, 14.3\% developed visual impairment and $5.7 \%$ developed blindness.

Conclusion-This study demonstrates that leprosy related ocular pathology progresses in some patients even after they are cured mycobiologically. The progressive leprosy related lesions are the result of chronic nerve damage; ocular lesions due to infiltration by Mycobacterium leprae did not develop. Based on the factors found to be associated with development of the most visually significant findings (posterior synechia, keratitis, and cataract) certain patients should be targeted at discharge for active follow up eye care. We suggest that patients with lagophthalmos (even in gentle closure), trichiasis, small pupils, and posterior synechiae should be screened regularly for the development of lagophthalmos in forced closure, keratitis, and cataract. (Br F Ophthalmol 2000;84:817-821)

There are approximately 1.5 million leprosy patients taking multidrug therapy (MDT), the World Health Organisation's recommended treatment regimen for leprosy. Another $10 \mathrm{mil}-$ lion have completed and been released from treatment. It is estimated that $1.5-2 \%$ of the total are blind from leprosy related causes and another $2 \%$ are blind from non-leprosy causes, chiefly age related cataract. ${ }^{1}$ The most common leprosy related causes of blindness are corneal disease secondary to lagophthalmos and "chronic iritis," which is an incompletely understood condition unique to leprosy that results in extreme miosis, posterior synechiae, iris atrophy, and, often, complicated cataract. In addition, older leprosy patients develop age related cataract with at least the same frequency as the non-leprosy population, although they rarely have access to the same eyecare services.

Although patients who have completed treatment are considered cured (because most of them are microbiologically negative) they still have many disabilities which were present before treatment began. In addition, they may have progressive disability because pre-existing nerve damage is not reversed by treatment. Thus, it is possible that after leprosy cure, new ocular pathology may develop. There is currently little information about the magnitude and nature of new pathology in cured leprosy patients. This is important from two different perspectives. One is to increase our understanding of the pathophysiology of ocular involvement in leprosy; the other is to provide information that would be useful in developing programmes to address the eyecare needs of leprosy patients who have been released from treatment. In most settings, once patients are released from treatment they are generally lost to follow up with the medical system and have little or no access to care. 
We studied the incidence of leprosy related ocular pathology and cataract during an 11 year follow up period in patients living in leprosy resettlement villages who had been released from treatment.

\section{Methods}

STUDY SITE AND POPULATION

The study was undertaken in central South Korea. Leprosy is no longer a public health problem in South Korea; only 39 new cases of leprosy (0.1 per 100000 population) were registered in 1998 and there are approximately 18800 patients who have been treated and released. Treated and released patients are divided between domiciliary (53\%), resettlement village $(36 \%)$, and leprosaria $(11 \%)$. The healthcare infrastructure in South Korea is well developed and includes all medical specialties. All leprosy patients (cured or on MDT) are included in the government provided free healthcare programme. Numerous nongovernmental hospitals also provide health care free of charge. Eye care for leprosy patients has been available through the Catholic Skin Clinic and Hospital, Taegu (full time ophthalmologist since 1989), Yosu Aeyeong Hospital, Yosu (visiting ophthalmologists), and the Korean Leprosy Institute, Anyang (visiting ophthalmologists).

This study was carried out through the Catholic Skin Clinic and Hospital (CSCH), which is responsible for eight leprosy resettlement villages in the Taegu area.

During the months of October and November 1988 standardised eye examinations were performed on 501 (83\%) of the 605 patients in the eight villages under the care of the $\mathrm{CSCH}$. All villagers were encouraged to come for examination in a central meeting point in the village. At least two trips were made to each village and attempts were made to find villagers who did not come and to see them in their homes if possible. Ophthalmological studies with these patients have been described previously. $^{2-5}$ In 1989, following the baseline assessment and initiation of a health worker training programme, the $\mathrm{CSCH}$ chose to recruit a full time ophthalmologist. Since then the CSCH has provided cataract, lagophthalmos and, to a lesser extent, trichiasis surgery to leprosy patients from throughout South Korea. These patients were traced in 1995 to determine mortality associated with blindness. ${ }^{5}$ In May 1999 standardised eye examinations were repeated in this population.

Visual acuity was measured with an illuminated tumbling $\mathrm{E}$ chart by a trained examiner. Clinical examinations in 1988 were performed by two examiners (SL and Dr Lee Ho-Sung) in a semidarkened room, using a torch to examine the ocular adnexae and a portable slit lamp for the cornea (with fluorescein instillation). Corneal sensation was determined by the reaction to a cotton wisp introduced from below. A Perkins applanation tonometer was used in alternate patients to measure intraocular pressure, both in the upright and then the supine positions. Dilatation and fundus examination were performed if necessary and to assess cause of low vision. Examinations in 1999 were performed in the same rooms by two examiners (SL and NT) using the same equipment and in the same fashion except that all patients had upright IOP measurement; no supine IOP measures were taken.

The following were recorded: visual acuity, blink pattern (observed while the patient was unaware of being examined and graded abnormal when there was more than 15 seconds between blinks or if the blink was incomplete); lagophthalmos (in both gentle and forced closure); pupil size, shape, and reaction to light; presence of corneal opacity and keratitis; conjunctival injection; trichiasis; anterior chamber clarity; presence of posterior synechia; lens clarity; and upright and supine (1988 only) intraocular pressure. If there was any question about synechia or lens clarity, mydriatic drops were instilled and the patient was re-examined. Cataract was defined as the presence of lens opacity judged to be consistent with a corrected visual acuity of $6 / 18$ or worse. Aphakia or pseudophakia was also included as evidence of incident cataract. In both 1988 and 1999, a sample of $10 \%$ of patients were examined by both examiners to ensure agreement.

Clinic records were reviewed, and demographics (age, sex, disease duration) and non-ocular clinical data (relapse in intervening periods, disease type, PGL-1 antibody levels) was abstracted.

STATISTICAL ANALYSES

Incidence of ocular pathology was calculated from those patients who did not have the specific finding in 1988 . We considered primary leprosy related ocular findings to include lagophthalmos (either on gentle or forced closure), posterior synechia, or keratitis. We used proportional hazards regression (forward stepwise) to analyse occurrence of specific findings according to demographic and clinical characteristics associated $(p<0.05)$ with pathology by univariate analysis. Relative risks (95\% CI) were generated. Our findings are reported by patient rather than by eye. We excluded patients who had died, moved away, or refused examination from our analyses.

\section{Results}

Among the 501 patients examined in 1988, $270(53.9 \%)$ were followed up in 1999 . Of the patients lost to follow up, there were 84 (16.8\%) deaths; 40 (7\%) patients moved away, and $107(21.4 \%)$ patients refused examination or were absent from the village at the time of examination. Participation varied widely by village, participation was lowest in the largest village, and was not significantly different in 1999 compared with 1988. Demographic information on the study population is given in Table 1. As described in a previous publication, patients who were blind in 1988 had a 4.8-fold excess risk of death compared with their non-blind same age peers. ${ }^{5}$ Inclusion of deaths between 1995 and 1998 did not change this association. Consequently, blindness was less common among patients examined in 
Table 1 Demographic characteristics of study population

\begin{tabular}{lllll}
\hline \multicolumn{5}{c}{1999 study population } \\
\cline { 2 - 5 } & $\begin{array}{l}\text { Examined } \\
(n=270)\end{array}$ & $\begin{array}{l}\text { Refused } \\
(n=107)\end{array}$ & $\begin{array}{l}\text { Died } \\
(n=84)\end{array}$ & $\begin{array}{l}\text { Moved } \\
(n=40)\end{array}$ \\
\hline $\begin{array}{l}\text { Mean age in 1988 (SD) } \\
\text { Sex }\end{array}$ & $52.9(8.4)$ & $48.9(10.1)$ & $59.9(10.4)$ & $53.5(11.8)$ \\
$\quad \begin{array}{l}\text { Males } \\
\text { Females }\end{array}$ & $46.6 \%$ & $49.0 \%$ & $66.1 \%$ & $50 \%$ \\
$\begin{array}{l}\text { Mean duration of disease in } \\
\text { years in 1988 (SD) }\end{array}$ & $53.4 \%$ & $51.0 \%$ & $33.9 \%$ & $50 \%$ \\
$\begin{array}{l}\text { Type of disease } \\
\quad \text { Multibacillary }\end{array}$ & $44.2(7.9)$ & $30.4(9.3)$ & $87.3(10.8)$ & $34.1(10.0)$ \\
$\quad$ Paucibacillary & $79.9 \%$ & $73.1 \%$ & $76.0 \%$ & $76.7 \%$ \\
\hline
\end{tabular}

Table 2 Ocular pathology incidence in 11 year period $(n=270)$

\begin{tabular}{lll}
\hline & $\begin{array}{l}\text { Prevalence } 1988-99 \\
\%(95 \% \text { CI) }\end{array}$ & $\begin{array}{l}\text { Cumulative incidence } \\
\%(95 \% \text { CI })\end{array}$ \\
\hline $\begin{array}{l}\text { Lid changes } \\
\text { Abnormal blink pattern }\end{array}$ & $24.8( \pm 5.2)$ & $11.8( \pm 4.4)$ \\
Inability to close (gentle) & $28.5( \pm 5.4)$ & $3.6( \pm 2.6)$ \\
Inability to close (forced) & $21.5( \pm 4.9)$ & $2.4( \pm 2.1)$ \\
Trichiasis & $7.8( \pm 3.2)$ & $9.6( \pm 3.7)$ \\
Corneal changes & $6.7( \pm 3.0)$ & $17.5( \pm 4.7)$ \\
$\begin{array}{l}\text { Cornosure keratitis } \\
\text { Reduced corneal (visually disabling only) }\end{array}$ & $3.3( \pm 2.1)$ & $2.4( \pm 1.9)$ \\
Uveal changes & $13.3( \pm 4.1)$ & $20.1( \pm 5.1)$ \\
Miotic pupil (<2 mm) & $20.1( \pm 4.8)$ & $4.3( \pm 2.7)$ \\
Abnormal pupil reaction & $24.2( \pm 5.2)$ & $5.5( \pm 3.2)$ \\
Posterior synechia & $18.1( \pm 4.6)$ & $7.0( \pm 3.4)$ \\
Lens changes & $10.4( \pm 3.7)$ & $26.4( \pm 5.7)$ \\
\hline Cataract (vision reducing) & &
\end{tabular}

Table 3 Risk of incident synechia, keratitis, and cataract

\begin{tabular}{|c|c|c|}
\hline & Unadjusted RR (95\% CI) & Adjusted RR (95\% CI) \\
\hline \multicolumn{3}{|l|}{ Risk of incident synechia } \\
\hline Age & $1.07(1.04-1.11)^{\star}$ & $1.02(0.96-1.08)$ \\
\hline Duration of leprosy & $1.09(1.05-1.13)^{\star}$ & $1.03(0.97-1.09)$ \\
\hline Pupil size $(<2 \mathrm{~mm})$ & $10.72(4.46-25.77)^{\star \star}$ & $8.18(4.79-13.95)^{\star \star}$ \\
\hline \multicolumn{3}{|l|}{ Risk of incident keratitis } \\
\hline Abnormal blink & $5.66(3.38-9.49)^{\star \star}$ & $1.41(0.86-2.33)$ \\
\hline Lagophthalmos (gentle closure) & $7.76(4.35-13.83)^{\star \star}$ & $7.46(5.36-10.39)^{\star \star}$ \\
\hline Poor corneal sensation & $3.01(1.75-5.19)^{\star \star}$ & $1.77(1.22-2.57)$ \\
\hline Disease type (paucibacillary) & $2.34(1.38-3.98)^{\star}$ & $1.60(1.16-2.22)$ \\
\hline Trichiasis & $2.31(1.20-4.46)^{\star}$ & $2.21(1.46-3.34)^{\star}$ \\
\hline \multicolumn{3}{|l|}{ Risk of incident cataract } \\
\hline Age & $1.08(1.06-1.09)^{\star}$ & $1.07(1.05-1.08)^{\star}$ \\
\hline Disease duration & $1.07(1.05-1.09)^{\star}$ & $1.02(0.99-1.04)$ \\
\hline Pupil reaction & $2.79(1.88-4.13)^{\star}$ & $1.23(0.60-2.59)$ \\
\hline Pupil size $(<2 \mathrm{~mm})$ & $3.03(2.06-4.44)^{\star}$ & $2.41(1.83-3.18)^{\star \star}$ \\
\hline Posterior synechia & $2.26(1.48-3.43)^{\star}$ & $1.27(0.86-1.89)$ \\
\hline
\end{tabular}

$\mathrm{RR}=$ relative risk, $\mathrm{CI}=$ confidence interval, ${ }^{\star} \mathrm{p}<0.01,{ }^{\star \star} \mathrm{p}<0.001$.

1999 than in 1988 . The patients who accepted examination in 1999 and those who refused in 1999 were not different with respect to vision, lagophthalmos, trichiasis, posterior synechia, and cataract in 1988.

INCIDENCE OF LEPROSY RELATED PATHOLOGICAL LESIONS

A large proportion of these cured patients already had potentially blinding ocular pathology in 1988 (Table 2). Cumulative incidence of leprosy related ocular pathology ranged as high as $20.1 \%$ (reduced corneal sensation). All posterior synechia cases were among multibacillary patients; excluding paucibacillary patients from our analysis reveals a cumulative incidence for posterior synechia of $9.3 \%$ (95\% CI: $4.8-13.8 \%$ ). Univariate analysis demonstrated that the development of posterior synechia (in the 11 year period) was associated with age, duration of disease, and small pupil size (usually accompanied by poor pupil reaction); regression analysis revealed that only size of
Table 4 Incidence of primary leprosy related pathology

\begin{tabular}{lll}
\hline & $N o$ & $\%^{\star}(S D)$ \\
\hline Keratitis & 10 & $6.4(3.8)$ \\
Synechia & 11 & $7.1(4.0)$ \\
Lagophthalmos & 6 & $3.8(3.0)$ \\
Any of the three & 23 & $14.7(5.6)$ \\
\hline$\star(n=156$ without pathology in 1988).
\end{tabular}

pupil ( $<2 \mathrm{~mm}$ ) was independently predictive of incident synechia (Table 3 ).

The only factor associated with incident lagophthalmos on forced closure was lagophthalmos on gentle closure. Among the 19 cases of lagophthalmos on gentle closure in 1988, three $(15.8 \%)$ progressed to lagophthalmos on forced closure. No factors were associated with the development of lagophthalmos on gentle closure. Univariate analysis revealed that incident keratitis was associated with the presence of lagophthalmos (even on gentle closure), inadequate blink, type of disease, reduced corneal sensation, and trichiasis. Proportional hazards modelling showed that only lagophthalmos (gentle closure) and trichiasis were independently associated with keratitis (Table 3). In none of the trichiasis cases was there any entropion.

As pre-existing leprosy related ocular pathology was associated with the development of additional ocular disease, we divided patients into two groups: patients in whom there was primary leprosy related ocular disease (defined as lagophthalmos, keratitis, or posterior synechia) in $1988(n=114)$ and patients in whom none of these conditions was found $(n=156)$. Among the patients in whom there was no primary leprosy related ocular disease, 23 $(14.7 \%)$ developed one or more of these conditions (Table 4). Incident pathology was more common in multibacillary patients $(16.7 \%)$ than paucibacillary patients $(6.9 \%)$ and more common in women $(17.4 \%)$ than men $(10.9 \%)$, although these differences were not statistically significant $(\mathrm{p}>0.05)$.

\section{CUMULATIVE INCIDENCE OF CATARACT}

Cataract was the most common ocular condition in these patients. Overall, among those with no vision reducing cataract in 1988, $26.4 \%$ of patients had developed a vision reducing lens opacity $(n=58)$ or had evidence of cataract extraction $(n=3)$. Univariate analysis revealed that age, duration of disease, pupil reaction, pupil size, and posterior synechia were associated with incident cataract (Table 3). Age and pupil size were independently associated with the development of cataract. Among the cataract cases $29.3 \%$ had preexisting posterior synechia; including individuals who developed posterior synechia in the intervening 11 years, this increased to $34.5 \%$.

CHANGES IN VISION

Among patients examined in both 1988 and 1999,35 patients $(14.3 \%$ of those who had vision $\geqslant 6 / 18$ in 1988) developed visual impairment (6/18-6/60) and 15 patients $(5.7 \%$ of patients who had vision $\geqslant 6 / 60$ in 1988) 
Table 5 Prevalence and incidence of visual impairment and blindness

\begin{tabular}{lll}
\hline & $\begin{array}{l}\text { Prevalence (1988) } \\
\text { No (\%) } 95 \% C I\end{array}$ & $\begin{array}{l}\text { Cumulative incidence } \\
\text { No(\%) } 95 \% C I\end{array}$ \\
\hline $\begin{array}{l}\text { Presenting visual acuity better eye } \\
\text { Blind (<6/60) }\end{array}$ & $7(2.6) \pm 1.9$ & $15 / 263(5.7) \pm 2.8$ \\
$\begin{array}{l}\text { Visually impaired (6/24-6/60) } \\
\text { Normal vision (>6/18) }\end{array}$ & $18(6.7) \pm 3.0$ & $35 / 245(14.3) \pm 4.4$ \\
$\begin{array}{l}\text { Presenting visual acuity worse eye } \\
\text { Blind }(<6 / 60)\end{array}$ & $245(90.7) \pm 3.5$ & \\
$\begin{array}{l}\text { Visually impaired (6/24-6/60) } \\
\text { Normal }(>6 / 18)\end{array}$ & $46(17.0) \pm 4.5$ & $26 / 224(11.6) \pm 4.2$ \\
& $31(11.5) \pm 3.8$ & $41 / 193(21.2) \pm 5.8$ \\
\hline
\end{tabular}

${ }^{\star}$ This represents patients who have become blind or visually impaired in the 11 year period.

Table 6 Causes of incident visual impairment and blindness

\begin{tabular}{lll}
\hline & $\begin{array}{l}\text { Cataract or uncorrected aphakia } \\
\text { No (\%) }\end{array}$ & $\begin{array}{l}\text { Cornea or other } \\
\text { No (\%) }\end{array}$ \\
\hline $\begin{array}{l}\text { Better eye } \\
\text { Incident blind }\end{array}$ & $13(86.7)$ & $2(13.3)$ \\
Incident visually impaired & $28(80.0)$ & $7(20.0)$ \\
Worse eye & $21(80.8)$ & $5(19.2)$ \\
Incident blind & $18(43.9)$ & $23(56.1)$ \\
Incident visually impaired & & \\
\hline
\end{tabular}

developed blindness (Table 5) Consideration of the worse eye (thus, including unilateral vision loss) revealed that $21.2 \%$ of patients developed vision loss in one or both eyes and $11.6 \%$ of patients developed blindness in one or both eyes. Most of the blindness and visual impairment was due to cataract (Table 6).

\section{Discussion}

This study demonstrates that leprosy related ocular pathology progresses in some patients after they are cured mycobacteriologically. Although follow up examinations were not possible on all patients, those who accepted follow up were not different (demographically or in terms of pre-existing pathology) from those who were not examined.

PATHOPHYSIOLOGY OF OCULAR COMPLICATIONS Among the incident lesions, an abnormal blink pattern, lagophthalmos, and reduced corneal sensation are due directly to nerve damage; these may all contribute to the development of keratitis. Pupil abnormalities and synechiae are part of the so called "chronic iritis" of leprosy which has been suggested to be due, at least in part, to sympathetic nerve damage. Thus, all of the progressive leprosy related lesions, except trichiasis, are the result of chronic nerve damage. Ocular lesions due to infiltration by Mycobacterium leprae (for example, iris pearls, limbal nodules) did not develop in this population. The low incidence of lagophthalmos and our sample size provided inadequate numbers for determining factors associated with incidence.

The finding of $9.6 \%$ incident trichiasis cases was unexpected. Trichiasis in leprosy may be due to loss of support of lash follicles secondary to infiltration by $M$ leprae. ${ }^{6}$ However, a recent report demonstrates that eyelash ptosis, associated with laxity in the pretarsal tissues, may be the underlying cause of the trichiasis in many leprosy patients. ${ }^{7}$ Trichiasis due to infiltration of the lash follicle by $M$ lep- rae would seem unlikely to progress in patients who have been bacteriologically negative for many years. On the other hand, eyelash ptosis might progress in patients with orbicularis weakness, leading to trichiasis.

PROGRAMME IMPLICATIONS

From the perspective of the leprosy control programme, these data has several implications. Of the 270 patients examined in both time periods $94(34.8 \%)$ had at least one leprosy related ocular lesion (lagophthalmos, keratitis, or posterior synechia) at baseline. Among those who did not have ocular lesions at baseline $15 \%$ developed one or more of these lesions over the 11 years of study (annual increase of $1.3 \%$ ).

The relatively low cumulative incidence of visual impairment $(14.3 \%)$ or blindness $(5.7 \%)$ may not be representative of the true incidence of visual impairment or blindness since blind patients have a significantly higher risk of death compared with their non-blind same age peers. ${ }^{5}$ Blindness and visual impairment occurred despite the presence of a well established high quality eye care infrastructure and the high cumulative incidence of vision loss and blindness in the worse eye is indicative of a failure in this population to use the services.

Based on the factors found to be associated with development of the most visually significant findings (posterior synechia, keratitis, and cataract) it would be advisable to target certain patients at discharge for active follow up eye care. We suggest that patients with lagophthalmos (even in gentle closure), trichiasis, small $(<2 \mathrm{~mm})$ pupils, and posterior synechiae should be screened regularly for the development of lagophthalmos in forced closure, development of keratitis, and significant cataract. In our population, this would include 131 patients $(48.5 \%$ of total patients) needing active follow up.

Over the 11 year period, cataract was responsible for blindness in $86.7 \%$ of patients (three had surgery but remained blind) and visual impairment in $80 \%$ of patients (three had surgery but were visually impaired). The exact contribution of leprosy to cataract development is not clear but it is estimated that the presence of leprosy triples the risk of cataract. ${ }^{8}$ The large number of patients who develop cataract emphasises the need to collaborate with blindness prevention programmes in order to provide necessary services to this population. If we assume that the cataract and lagophthalmos surgery performed in this population either cured or prevented visual disability and blindness, then it is clear that the number of patients who will have progressive visual loss is even higher than what we have demonstrated. Although leprosy programmes may be able to provide some lagophthalmos surgery services, only collaboration with ophthalmological services will meet the needs of the cataract blind.

Although the populations studied vary in a number of ways (for example, race, access to and uptake of ophthalmological services), our findings are in general agreement with a study 
in Holland ${ }^{9}$ which reported that lagophthalmos, corneal disease, and cataract comprise the important lesions which may progress in cured leprosy patients.

The patients in our study had all been treated with MDT, but most had also received dapsone in the era before MDT. This reflects the situation for most of the world's cured leprosy patients and our findings should be applicable to these patients. There will, however, be a growing number of cured patients in the future who will have been treated only with MDT from first diagnosis. It is not known yet whether MDT only patients will progress in the same way as those patients who had dapsone before MDT.

This study was supported in part by LEPRA (British Leprosy Relief Association) to whom we are grateful for support. We Relief Association) to whom we are grateful for support. We
have had invaluable support from Mrs Pak Ok-Hyeon and the staff at the Catholic Skin Clinic and Hospital.
1 Courtright P, Lewallen S. Ocular manifestations of leprosy. In: Johnson GJ, Minassian DC, Weale R, eds. The epidemiology of eye disease. London: Chapman and Hall, 1998.

2 Lewallen S, Courtright P, Lee HS. Ocular autonomic dysfunction and intraocular pressure in leprosy. $\mathrm{Br} \mathcal{F} \mathrm{Oph}$ thalmol 1989;73:946-9.

3 Courtright P, Lewallen S, Lee HS. Comparison of the old and new WHO leprosy disability grading scheme for ocular disabilities. Int Ophthalmol 1991;15:295-8.

4 Courtright P, Lee HS, Lewallen S. Training for primary eye care in leprosy. Bull World Health Organ 1990;68 $347-51$

5 Courtright P, Kim SH, Lee HS, et al. Excess mortality associated with blindness in leprosy patients in Korea. Leprosy Rev 1997;68:326-30.

6 Brand ME, ffytche TJ. Eye complications of leprosy. In: Hastings RC, ed. Leprosy. Edinburgh: Churchill Livingstone, 1985:224.

7 Guimaraes FC, Augusto A, Cruz V. Eyelid changes in longstanding leprosy. Ophthalmic Plastics Reconstruct Surg 1998; 14:239-43.

8 Waddel KM, Saunderson PR. Is leprosy blindness avoidable? The effect of disease type, duration, and treatment on eye damage from leprosy in Uganda. $\mathrm{Br} f$ Ophthalmol 1995;79:250-6.

9 Hogeweg M, Faber WR. Progression of eye lesions in leprosy: ten-year follow-up study in the Netherlands. Int $\mathcal{F}$ Leprosy 1991;59:392-7.

\section{Cover illustration: In the pink}

The greater flamingo (Phoenicopterus ruber) is a large bird and a member of the Phoenicopteridae family. This family is widespread with five separate species, including both old and new world flamingos. This species may stand as tall as six feet, and all have the long legs and flexible necks typical of wading birds. Probably related to herons, spoonbills, and ibises, they have unusual hooked-shaped bills allowing them to feed by dipping their mandibles under water and scooping backwards with their heads upside down. Commonly seen on the salt pans and shallow soda lakes of Africa, these birds have enthralled people from emperors to peasants for thousands of years. The birds live in large colonies and may live up to 50 years. Like many birds, flamingos are monogamous throughout their life. Flamingos will breed on small hillocks situated in shallow standing water as a protection against predators. Some of these species are endangered because of the reduction of wetlands and habitat destruction.

All species are varying and beautiful shades of pink, but it is not an intrinsic coloration. Flamingos feed on small amphipods, polychaetes, small crustaceans, and related species of shrimp. The lovely pale pink of the body, tinged with vermilion along the wing edges, as seen in this photograph, results from the shrimps and other invertebrates that are consumed. If the shrimps are bred to be white without coloration, so the flamingo will become white too.

A flamingo typically feeds with its head upside down immersing the mandible (and sometimes the whole head) under water. The upper mandible is narrow and fits into the lower mandible much like the lid of a box. The edges of the mandible have transverse plates called lamelli, which are used to strain invertebrates from the water. As the flamingo walks forward with its head upside down, it filter feeds and sifts through stirred up sediment, using its thick, spine-covered tongue to sieve liquid through the mandible, leaving only the crustaceans.

Flamingos share with gulls, albatrosses, and shearwaters an interesting adaptation for vision. These birds all have an intraocular structure known as an infula. This is a single, linear, trough-like fovea which continues in a horizontal meridian nearly throughout the entire retina. Although humans have a concentration of photoreceptors in the horizontal meridian, the vague increase in photoreceptors in this area offers us little known advantage and may be evolutionary detritus. Although the purpose of this concentration of photoreceptors in this horizontal meridian is not well understood, it appears that the visual acuity of flamingos is in the range of our own and this horizontal band may presumably be used for protection against predation. This linear strip of sharply focused visual acuity would allow a flamingo to see an approaching bird from a considerable distance. Even though these birds do congregate in flocks, they are preyed upon by eagles and large hawks that hunt by isolating injured or diseased birds. When a flamingo feeds, its head is in such a position that the infula would be just above and parallel to the flat horizon of a shallow lake allowing for a panoramic view of the horizon for much of the $360^{\circ}$ surround. How these foveae are neurologically integrated is not known. Such an evolutionary variation in foveal construction must be a clever approach for protection of this species.-Dr Ivan Schwab, Sacramento, California, USA 